\title{
Exploring Complexities of T2DM: Insulin Signaling, Resistance and Deficiency, Metabolic Links, Sexual Dimorphism and Endocrine Disruptors
}

\author{
Vinod Nikhra* \\ Department of Medicine, India \\ *Corresponding author: Vinod Nikhra, Department of Medicine, Hindu Rao Hospital, India

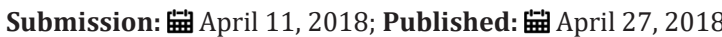

\begin{abstract}
Surging pandemicity of T2DM: The time-old and conventional wisdom holds that T2DM is a metabolic disease characterized by hyperglycemia due to a deficient action of insulin and that diabetic complications result due to high circulating glucose levels and glycation products. But, the characterization of T2DM with hyperglycemia is an overt simplification. T2DM is rather a complex interplay of metabolic alterations herald by hyperglycemia and it may be interesting to explore the complexities encompassing etiology, pathophysiology and genesis of T2DM and its complications.
\end{abstract}

Altered insulin homeostasis: In due course, in pre-diabetes and later in T2DM, the tissues develop resistance to the metabolic actions of insulin, culminating as decreased insulin action and hyperglycemia resulting from various factors. The IR is inherently variable and occurs at variable period in different body tissues, the major contributors to IR being obesity and physical inactivity.

The complexities of T2DM: The altered insulin signaling may be an important factor in the genesis of diabetic complications. The insulin sensitivity regulates $\beta$-cell function, and when glucose and lipids are increased for a long period, the $\beta$-cells function is compromised. The IR may seem to be deleterious in general, as it is associated with MetS, T2DM and allied metabolic disorders, but has certain protective and beneficial role. The pentosephosphate pathway is impaired due to thiamine deficiency and correction of thiamine deficiency by thiamine, restores IR and biochemical dysfunctions. Aging is the irreversible contributor to metabolic decline and related disorders, including T2DM. EDCs pose threats to metabolic homeostasis, being the paradox and a product of technological developments.

Conclusion-metabolic response and adaptations: The intensive treatment of T2DM with insulin may be harmful in obese patients who are unable to improve lifestyle to achieve a negative energy balance. The insulin-responsive tissues, such as the heart, skeletal muscle, adipose tissue, and the liver, are able to protect themselves from nutrient-induced damage by developing IR. Other tissues, such as nerves, eye, kidney and the vasculature, are less protected by IR. Here, the hypotheses relating to 'IR as adaptive defence mechanism' and 'insulin-induced metabolic stress' are attractive concepts and need to be explored further, in light of current research and new studies available.

Keywords: Metabolic syndrome; Type 2 diabetes mellitus; Insulin resistance; Insulin homeostasis; Thiamine deficiency; Endocrine disrupters; Gender related adiposity; Metabolic stress

Abbreviations: BMI: Body Mass Index; BPA: Bisphenol A; CVD: Cardio Vascular Disease; CNS: Central Nervous System; CoA: Coenzyme A; DPP-4: Dipeptidyl peptidase-4; DM: Diabetes Mellitus; T2DM: Type 2 Diabetes Mellitus; EDCs: Endocrine Disrupting Chemicals; ER: Endoplasmic Reticulum; GLUT: Glucose Transporter; DDT: Dichlorodiphenyltrichloroethane; FFA: Free Fatty Acids; GLP-1: Glucagon Like Peptide-1; POPs: Persistent Organic Pollutants; GDPH: Glyceraldehyde 3-Phos-Phate Dehydrogenase; GISS: Glucose Stimulated Insulin Secretion; NPY/AgRP: Neuropeptide Y/Agouti Related Peptide; IR: Insulin Resistance; SGLT2: Sodium-Glucose-Cotrans-Porter-2; MAPK: Mitogen-Activated Protein Kinase; GH: Growth Hormone; IGF: Insulin like Growth Factor; MetS: Metabolic Syndrome; NEFA: Non-Esterified Fatty Acids; NPY-Y1: Neuropeptide Y1 Receptor Y1; OHA: Oral Hypoglycaemic Agent; PVD: Peripheral Vascular Disease; POMC: Pro-Opiomelanocortin; PVN: Paraventricular Nucleus; PKC: Protein kinase C; PBDE: Poly Brominated Diphenyl Ether; TZDs: Thiazolidinediones; PCBs: Polychlorinated Biphenyl Ethers; PI3K/PKBAKT: Phosphoinositide-3-Kin-Ase/ Protein Kinase B (or) AKT; T1DM: Type 1 Diabetes Mellitus

\section{Introduction}

\section{Surging pandemicity of T2DM}

The time-old and conventional wisdom holds that type 2 diabetes mellitus (T2DM) is a metabolic disease characterized by hyperglycemia due to a deficient action of insulin and that diabetic complications result due to high circulating glucose levels and glycation products. The American Diabetes Association (ADA) has defined Diabetes Mellitus (DM) as, 'a group of metabolic diseases characterized by hyperglycemia resulting from defects in insulin secretion, insulin action, or both' [1]. The characterization of T2DM 
with hyperglycemia or the definition by ADA may seem an overt simplification. Rather, than a simple disease, T2DM is complex interplay of metabolic alterations herald by hyperglycemia. In fact, the elevated blood glucose levels are only one facet of diabetes. In the current times, there is a surging pandemicity of insulin resistance (IR), metabolic syndrome (MetS), obesity and T2DM, all of which are metabolic disorders and complexly interrelated. Thus, it may be interesting to explore the complexities of T2DM encompassing its etiology and pathophysiology, and the basis and genesis of its complications [2].

The pandemic of metabolic diseases including obesity, MetS and T2DM during recent years, cannot be attributed only to genetic background and changes in diet, exercise and aging [3]. There is considerable evidence that other environmental factors, which include the metabolic disruptors, may contribute to the rapid surge of these metabolic diseases during last 3-4 decades. The metabolic disruptors are endocrine disruptors or endocrine disrupting chemicals (EDCs) that increase the susceptibility to metabolic diseases. The International Diabetes Federation estimates that 20-25\% of the world's adult population suffers from MetS, which is a complex condition characterized by IR, abdominal obesity, dyslipidemia, hypertension and hyperglycemia, and a risk factor for cardiovascular disease (CVD), T2DM, stroke, chronic kidney disease and cancers [4].

\section{Altered insulin homeostasis}

There are various metabolic effects of insulin. It facilitates glucose transport into the cells especially muscle and fat cells, promotes glucose storage as glycogen, inhibits hepatic glucose production, stimulates amino acid uptake and protein synthesis in muscles and liver, and inhibits breakdown of fat and protein. It, thus, streamlines carbohydrate homeostasis to regulate the blood glucose levels within a narrow physiological range. Apart from these metabolic effects, insulin exerts important regulatory actions on almost every tissue in the body. It regulates gene transcription and synthesis of various key enzymes, influences the cell cycle, and protects cells from apoptosis. In addition, insulin plays an important role in the central nervous system (CNS) and is involved in regulation of neuronal physiology and survival, neural proliferation, apoptosis and synaptic transmission. As documented by various studies, insulin plays a role in memory and cognition, and protects synapses from Alzheimer's linked toxins. But, in due course, in pre-diabetes and T2DM, the tissues develop resistance to the metabolic actions of insulin, culminating as decreased insulin action and increased blood glucose level resulting from decreased glucose transport into fat and muscle, decreased use of glucose by liver, muscles and other body organs, and increased gluconeogenesis. But, the IR is variable in different body tissues; some develop IR early in course of disease, whereas others may retain effectivity to insulin action.

The IR and central obesity are significant contributors for MetS, the prevalence which is increasing along with the increase in obesity and has reached an epidemic proportions affecting between $24 \%$ and $34 \%$ of the adult US population [5]. The major contributors to IR are obesity and physical inactivity (Figure 1). Obesity, especially excess fat around waist and complex interactions in the adipose tissue draw immune cells to the area to trigger low-level chronic inflammatory state, and is a primary cause of IR.

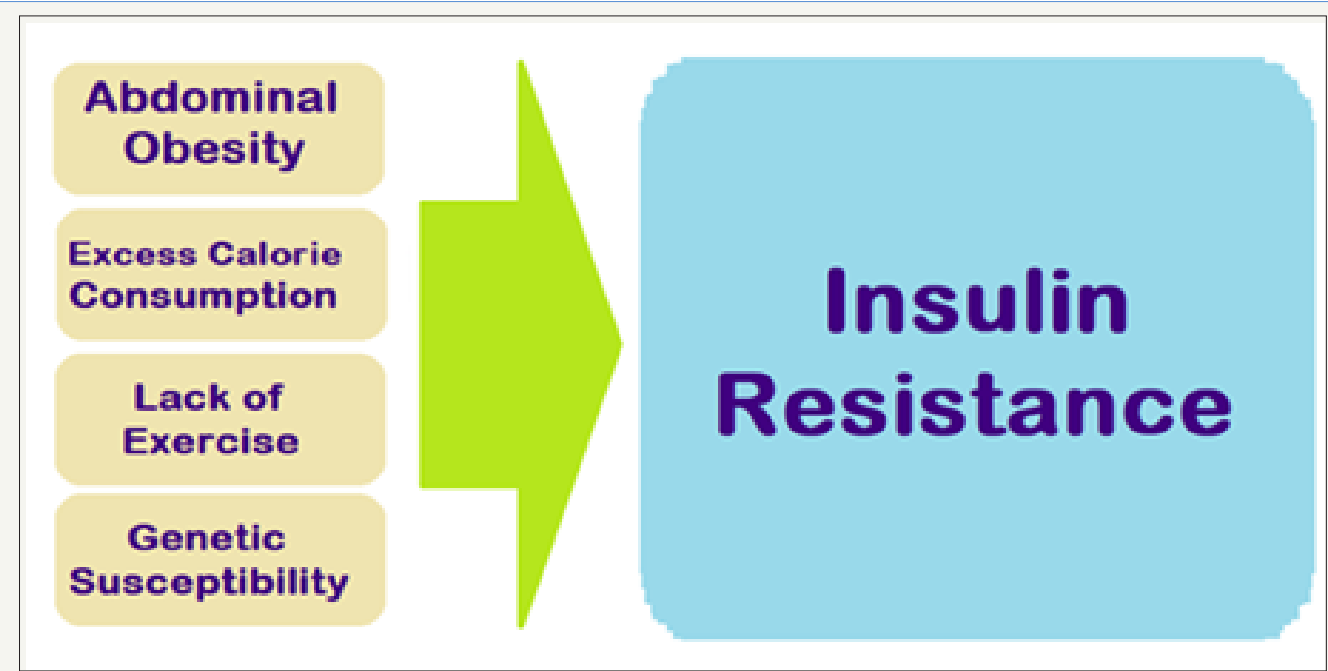

Figure 1: General causes of insulin resistance.

The adipose tissue produces hormones and other substances that can cause IR, MetS, T2DM, hypertension and CVD. Physical in activity is associated with insulin resistance, often leading to T2DMand obesity. In the body, more glucose is used by muscle than other tissues. Studies show that following exercise, the muscles become more sensitive to insulin, reversing IR and lowering blood glucose levels. Exercise also helps muscles absorb more glucose without the need for insulin. T2DM and MetS is often causally linked to marked changes in diet and lifestyle, namely increase in dietary intake of high calorie diets and a concomitant reduction in the physical activity level [6]. 


\section{The compexities of T2DM}

T2DM is a complex disease, involving a multitude of organs and systems, and having various metabolic and non-metabolic manifestations. The studies suggests that the altered insulin signaling may be an important factor in the genesis of diabetic complications and the decline in non-metabolic effects of insulin has been linked to the development of diabetic complications. The diabetic complications include delayed wound healing, peripheral neuropathy, hypertension, risk of heart disease and stroke, nephropathy and sexual dysfunction. The periodontal disease and peripheral vascular disease (PVD) result from hyperglycemia as well as altered fibroblast activity. As proven experimentally, the fibroblasts isolated from diabetic patients have decreased proliferation and increased apoptosis. This has been related to the delayed wound healing and PVD culminating into gangrene. Insulin has been shown to relax blood vessels, and the loss of this effect due to insulin deficiency has been related to cause hypertension. The T2DM patients are susceptible to infection and have reduced ability to deal with infection due to deficient insulin action. The lack of the insulin's protective role in the CNS, due to deficiency of insulin appears to be a major factor for the CNS complications of diabetes, and research has documented that diabetes impairs hippocampus dependent memory, synaptic plasticity and neurogenesis. Simultaneously, T2DM and IR are associated with increased risk of developing Alzheimer's disease.

\section{Genesis and after math-the IR}

The IR is a state when a given concentration of insulin produces a less-than-expected physiological effect. The causes of IR can be the pre-receptor including abnormal insulin and anti-insulin antibodies, at the receptor including decreased number of receptors, reduced binding of insulin, insulin receptor mutations and insulin receptor-blocking antibodies, and the post-receptor which include the defective signal transduction and mutations of GLUT4. Various combinations of the causes are common, for example, in obesity, the IR is associated with post-receptor abnormality and a decreased number of insulin receptors, and aging causes IR mainly through a decreased production of GLUT-4. There is a significant relationship between IR and CV risk factors, especially observed in middleaged persons. Further, IR is an established independent predictor of a range of disorders and the resistance to insulin sets in long before any disease signs start appearing [7]. The insulin sensitivity regulates $\beta$-cell function, and the insulin resistant subjects, whether they are obese or lean, have greater insulin secretory response and a lower insulin clearance than insulin-sensitive individuals leading to higher serum insulin levels. However, when glucose and lipids are increased for a long period, the $\beta$-cells function is compromised, leading to the onset of T2DM.

It appears that the ability of pancreatic $\beta$-cells to integrate response to changes in insulin sensitivity involves increased metabolism and metabolic signals including signaling molecules from adipocytes as well as and fatty acyl-Co As that augment insulin release via the exocytosis and protein kinase C (PKC). The alterations in the pancreatic $\alpha$-cells function, which are responsible for glucagon production and release, also contribute to T2DM [8]. The mass of pancreatic $\alpha$-cells does not decrease in T2DM, as opposed to the $\beta$-cells, resulting in an increased $\alpha$-to- $\beta$ cell ratio. Thus, when $\beta$-cells are healthy, their adaptive responses counterbalance IR and preserve normal glucose tolerance. However, when $\beta$-cell dys function sets in due to genetic causes, environmental perturbations, or both, then the subject is prone to develop impaired glucose tolerance, IR, high fasting glucose levels and T2DM.

\section{The paradox of IR}

The IR may seem to be deleterious, in general, as it is associated with MetS, T2DM and allied disorders. In fact, IR has certain beneficial role being a physiological adaptation to divert the excess nutrients from the susceptible tissues to adipose tissue for storage. Developmentally, IR has been preserved and its persistence all through the evolution of species, suggests that it benefits survival [9]. The IR protects against excess nutrient entry into cells which can cause glucolipotoxicity and contribute to mitochondrial dysfunction and endoplasmic reticulum stress [10]. IR, thus, regulates metabolic stress due high insulin levels in early T2DM and protects the susceptible tissues. Further, IR is important in various states such as starvation, immune activation, growth and cancer, to spare glucose for essential biosynthetic processes in a disturbed homeostasis milieu. The IR is, thus, essentially a defence mechanism to protect certain tissues from nutrient-induced injury [11].

Certain tissues like the endothelial cells are the directly exposed to the nutrients and hormones through the blood stream and pave a way to genesis of both micro- and macro vascular complications of diabetes $[12,13]$. The successful lowering of blood glucose and FFAs by exogenous insulin results in improved endothelial functioning due to a reduction in nutrient-induced IR of the PI3K/ AKT pathway [14]. But, the use of high doses of insulin therapy, in subjects who are refractory to its glucose-lowering effect, does not improve PI3K/AKT insulin signaling in the endothelium, rather has an increased harmful signaling through the MAPK pathway, increasing risk of atherosclerotic vascular complications [15]. Insulin signaling in endothelial cells can, via the PI3K, cause vasodilation and anti-thrombotic effect, and via the MAPK pathway, cause vasoconstriction and pro-thrombotic effect. In response to an excess nutrient supply, as occurs in poorly-controlled T2DM, selective IR in the PI3K pathway occurs leading to unopposed signaling through the MAPK pathway and increasing the risk of vascular events. If high exogenous insulin therapy is successful at improving blood nutrient levels, then insulin signaling through the PI3K pathway is beneficial to vascular health. In case the high dose exogenous insulin therapy fails to control blood nutrients, then the IR in the PI3K pathway is not relieved and insulin signaling predominates via the harmful MAPK pathway and increases the risk of vascular events. 
The metabolic abnormalities of T2DM result from insulin deficiency, primarily affecting metabolism in liver, muscle and adipose tissues. There is IR in pre-diabetes, metabolic syndrome and T2DM, which impairs the insulin action and its ability to metabolise glucose. Because of IR, the effectiveness of the phase 1 insulin response is lost. Instead, there is phase 2 insulin response, which is a delayed response, leading to higher post prandial blood sugar levels in T2DM and pre-diabetics. Also due to IR, the cells do not respond adequately to insulin for transporting glucose into the cells from the blood stream for which higher levels of insulin are required. Because of IR, the insulin is less effective and there is an increased insulin secretion from $\beta$-cells to compensate. Thus, insulin levels are high, especially early in the disease. In due course, the strain of producing more insulin harms the $\beta$-cells, which fail in due course and undergo apoptosis. The loss of $\beta$-cells further exacerbates hyperglycemia. In the disturbed homeostasis, hyperglycemia is caused by increased glucose production coupled with its decreased utilization. Simultaneously, hyperglycemia itself may impair insulin secretion, because high glucose levels desensitize $\beta$-cells and cause $\beta$-cells dysfunction. As a whole, the energy utilization, thus, shifts from carbohydrate catabolism to lipid oxidation, due to increased IR in peripheral tissues and decreased insulin production from $\beta$-cells.

There exists a paradox about physiological role of IR. In some ways, IR might actually serve as an adaptive mechanism in some tissues by preventing excess uptake of nutrients by cells. Indeed, differential IR of various organs can direct nutrient flux to adipose tissue, a factor that may have evolutionary benefits in regulating the nutrient storage. Moreover, IR has been proposed as an antioxidant defense mechanism [16]. In both impaired glucose tolerance and T2DM, tissues such as muscle, fat and liver, in general, become less responsive or resistant to insulin. This state is also associated with obesity, hyperlipidaemia, hypertension and atherosclerosis. The pathophysiology of IR involves a complex interplay of signalling pathways, linked to the insulin receptors and regulating intermediary and cellular metabolism [17]. Various other hormones as well as signalling events attenuate the insulin action, and are important in progression of metabolic abnormalities and complications of T2DM

\section{Aging, obesity and T2DM}

Aging and T2DM: Aging is the important and irreversible contributor to the metabolic decline and related disorders, including T2DM. The aging directly affects carbohydrate homeostasis by altering pancreatic $\beta$ cell function, $\beta$ cell mass, insulin secretion and insulin sensitivity, and predisposes for IR, impaired glucose tolerance and T2DM. Associated with the aging process, there occur changes in somatic composition including obesity and physiological decline in growth hormone (GH), insulin-like growth factor-1 (IGF1 ), and sex steroids. The prevalence of T2DM increases with age and peaks at 60-74, and about one third of the elderly have diabetes and three quarters have diabetes or prediabetes [18]. Collectively, these age-related alterations in metabolism and somatic fat distribution are active participants in a vicious cycle that can accelerate the aging process and the onset of metabolic disorders including T2DM [19].

(Modified from the fig in Review Article, Nikhra V. The Altered Metabolic Homeostasis in Middle Aged and Elderly: Aging and Other Determinants of Insulin Secretion, Action and Resistance. OAJ Gerontol\& Geriatric Med. 2018; 3(2): 555608).

With age (Figure 2), there is decreased glucose stimulated insulin secretion (GSIS), disorderly insulin release, decreased insulin pulse amplitudes, and decreased response to glucose oscillations as well as alterations in insulin clearance [20,21]. Many factors lead to decreased insulin secretion with age and include the age-associated loss of Sirt1-mediated GSIS [22], decreased $\beta$-cells sensitivity to circulating incretins, age-associated decrease in mitochondrial function and increased oxidative stress [23].

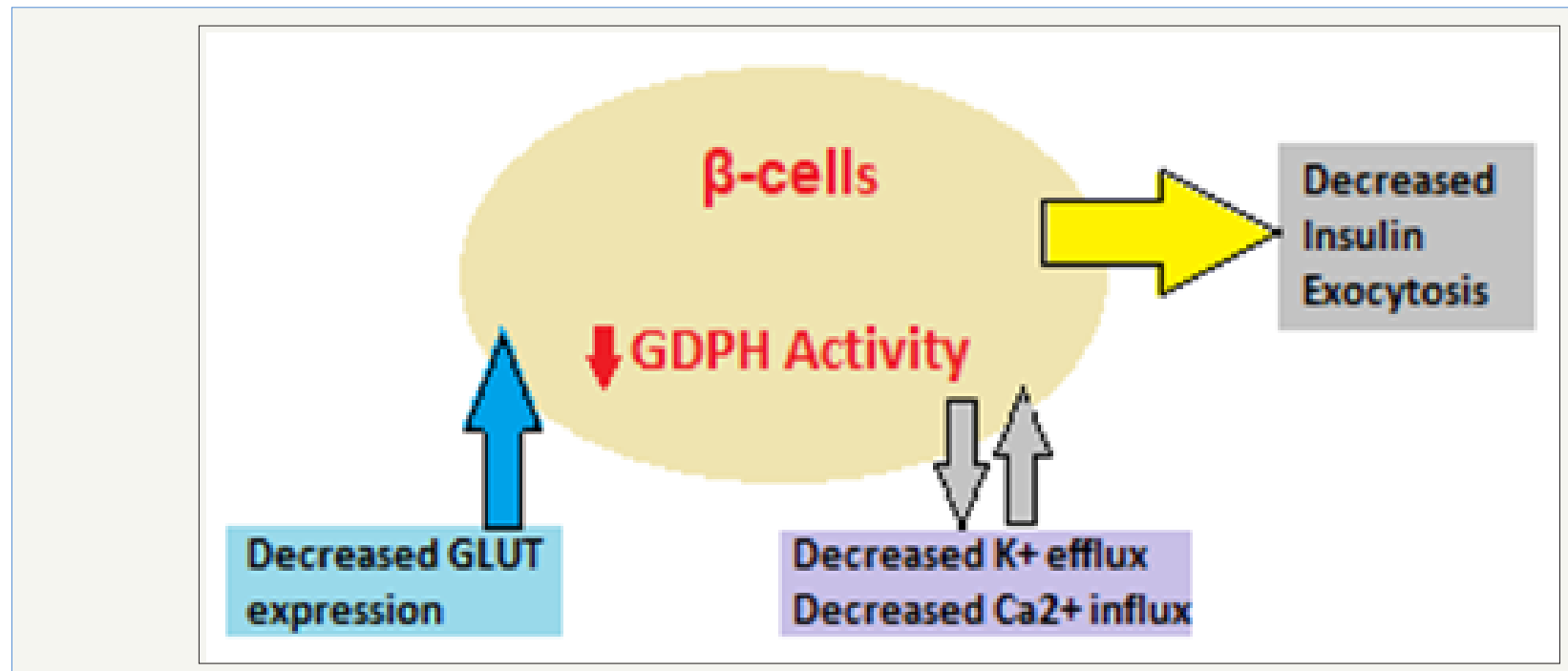

Figure 2: Impact of aging on insulin secretion leading to T2DM. 
The insulin secretory defects are superimposed on an increased insulin demand, IR, impaired glucose homeostasis and glucose intolerance. Further, aging is associated with a decline of insulin action, leading to high prevalence of impaired glucose tolerance and T2DM among the elderly. The initiation of the glucose transport is the first step that linked with insulin release from the $\beta$ cells. GLUT2 is the main transporter, but GLUT1 and GLUT3 are also important. The age-associated decrease in GLUT2 expression has been documented in aged rodent models [24]. The decrease in GDPH activity leads to decrease in GSIS. The exocytosis of insulin granules is the final step of insulin secretion. Calcium is the main regulator for exocytosis and since the net uptake of $\mathrm{Ca} 2+$ is decreased with age and it influences the insulin granule exocytosis.

Further, the $\beta$-cell mass and various aspects of functions decline with age, contributing to the age-associated defects in insulin secretion. The endoplasmic reticulum (ER) stress plays a role in the pathogenesis of diabetes by contributing to both pancreatic $\beta$-cell dysfunction and peripheral IR [25]. The aging-associated sedentary life style and diminished physical activity are other important factors for age-related changes of glucose homeostasis. The changes in somatic composition due to decreased lean body mass and contractile strength with age also contribute to the reduction in insulin stimulated glucose uptake.

Obesity and T2DM: Obesity is a major contributor to IR and MetS and associated T2DM. Most patients with T2DM are overweight or obese, and the global epidemic of obesity explains the dramatic increase in the incidence and prevalence of T2DM over the past 3 decades. There are intriguing connections between obesity and T2DM (Figure 3). Excess weight is an established risk factor for T2DM, yet most obese individuals do not develop T2DM. The studies indicate that influence of obesity on T2DM risk is determined by the degree of obesity as well as the sites of fat accumulation. The visceral adiposity, as reflected by increased abdominal girth or waist-to-hip ratio, is associated with MetS, T2DM and CVD. Apart from, the type of somatic fat distribution, various subtypes of adipose tissue are functionally distinct and affect glucose homeostasis differentially. The adipose tissue is composed of heterogeneous cell types, and adult humans have limited and variable numbers of brown fat cells playing a role in thermo genesis, white fat cells and Immune cells within adipose tissue which influence the metabolic processes.

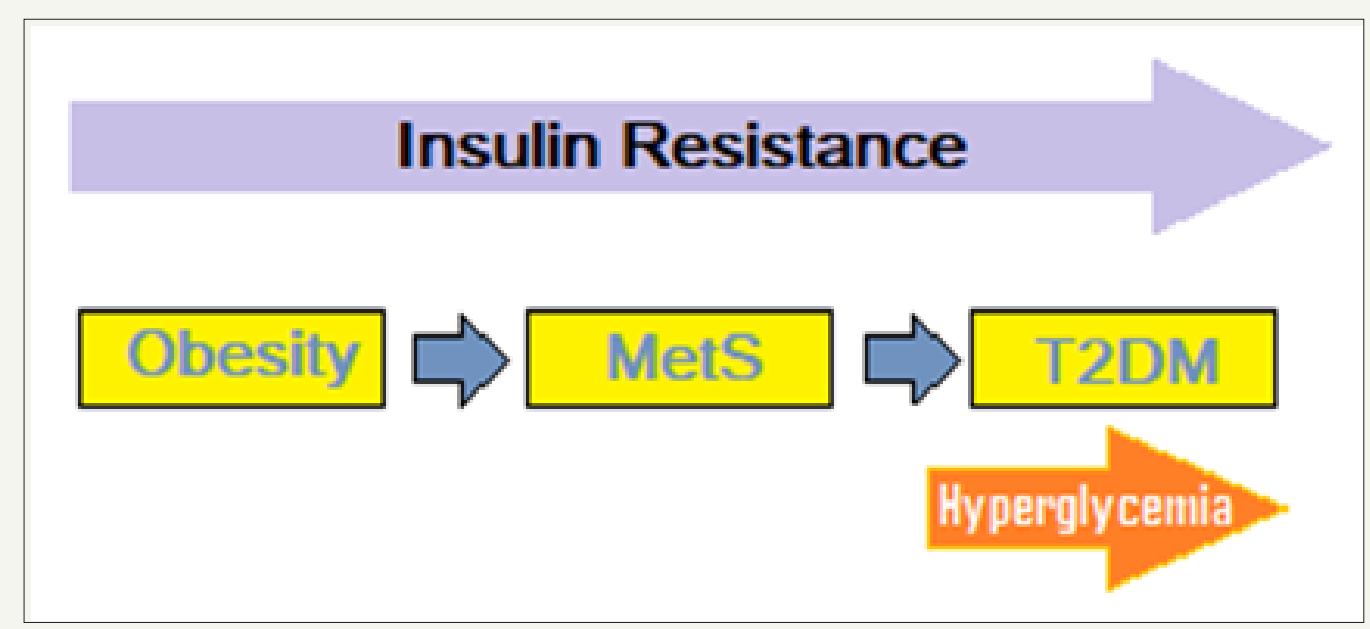

Figure 3: The obesity and T2DM links.

It is held that at least three distinct mechanisms link obesity to IR and predispose to T2DM. For one, the increased production of adipokines/cytokines, including tumor necrosis factor- $\alpha$, resistin, and retinol-binding protein 4 , that contribute to insulin resistance and the reduced levels of adiponectin [26]. Secondly, ectopic fat deposition, particularly in the liver and in skeletal muscle and its dis-metabolic effects; and finally, the mitochondrial dysfunction, evident by decreased mitochondrial mass and/or function and stressed endoplasmic reticulum (ER). Mitochondrial dysfunction is an important alteration linking obesity to T2DM by decreasing insulin sensitivity as well as compromising $\beta$-cell function [27]. The ER is stressed when it has more nutrients to process than it can handle and sends signals to down-grade the insulin receptors on the cell surface. It has been documented that obesity causes similar stress ER, which suppresses the insulin receptors, leading to IR. These facets of metabolic interactions are complex, with the relative impact of each inconstant.

There is a progressive $\beta$-cell dysfunction in obese individuals. The link between obesity and hyperinsulinemia, reflects compensation by insulin-secreting $\beta$-cells to systemic IR. Even the obese normoglycemic individuals have increased $\beta$-cell mass and function. Obesity-induced glucose intolerance reflects failure of the compensatory responses. Factors predisposing to $\beta$-cell decompensation is genetic as well as epigenetic [28]. Genetic studies have helped identified some key molecules related to $\beta$-cells homeostasis, which may be the potential mediators predisposing obese individuals to T2DM [29]. Although the link between obesity and T2DM involves two distinct phenomena - obesityinduced IR and $\beta$-cell failure, the 'unified field theory' underlines 
the possibility the two may share common underlying defects, which favour progressive weight gain and metabolic impairment contributing to $\beta$-cell decompensation. A possible link could be the sustained exposure to excess nutrient concentrations leading to deleterious cellular effects such as impaired inflammatory signaling, endoplasmic reticulum stress, excess production of reactive oxygen species, mitochondrial dysfunction, accumulation of triglycerides and/or fatty acyl intermediates, and activation of serine-threonine kinases [30]. Collectively, these responses contribute to the pathogenesis of IR in the liver, skeletal muscle, and adipose tissue, and may involve $\beta$-cells as well.

The reduced IR, in turn, worsens the nutrient excess problem by raising circulating concentrations of glucose, free fatty acids, and other nutrients. As a result, a vicious cycle is generated where obesity-induced nutrient excess triggers inflammatory responses that increase IR, placing a greater demand on the $\beta$-cell. In obesity, the non-esterified fatty acids (NEFAs), glycerol, hormones, cytokines, Proinflammatory markers, and other substances involved in the development of IR are also increased [31]. The elevated fatty acids in the plasma, decrease glucose transport into the muscle cells, as well as increased fat breakdown, leading to elevated hepatic glucose production. IR and pancreatic $\beta$-cell dysfunction occurring simultaneously lead to T2DM. The resulting abnormal glycemia contributes to micro vascular and metabolic complications. The IR with lipid abnormalities, thrombotic abnormalities, as well as atherosclerotic risk factors like smoking, family history, and hypertension potentiate the CV risk. The 'ticking clock' hypothesis of diabetic complications by Haffner et al. [32] outlined that the onset of hyperglycemia puts one at risk for developing macro vascular complications at an earlier point, perhaps at the onset of IR and before onset of hyperglycemia itself [32].

The brain neuro-circuits governing energy homeostasis also affect insulin sensitivity in the liver and other peripheral tissues. An inflammation similar to the one induced by obesity in peripheral insulin-sensitive tissues also occurs in the areas of the brain. The disturbed neuro-circuits may also contribute to deteriorating $\beta$-cell dysfunction. Equally challenging is the problem of weight regain, which usually follows any degree of weight loss. To explain this phenomenon, the current models of energy homeostasis predict genetic or acquired defects in key neuro-circuits that dampen the responses to adiposity-related humoral signals.

\section{T2DM and physical inactivity}

The BMI has a strong relationship to diabetes and IR, and the existence of several factors like increased prevalence of obesity among all age groups, physical inactivity, altered dietary habits and urbanization, adds to the increasing prevalence of IR and rising incidence of T2DM [33].

Further, the physical inactivity and over-nutrition have been linked to obesity, which is considered the most crucial factor in the development of metabolic diseases. The adipose tissue affects metabolism by secreting hormones, glycerol, and other substances including leptin, cytokines, adiponectin, proinflammatory substances, and by releasing NEFAs. In obese individuals, the secretion of these substances will be increased. The cornerstone factor affecting insulin insensitivity is the excess release of NEFAs, which is observed in T2DM and in obesity, and it is associated with IR [34].

Ceasing regular physical activity impairs glycemic control, suggesting that physical inactivity plays a key role in the development of T2DM [35]. The likelihood of having diabetes and diabetes-related cardiovascular comorbidities increased with BMI regardless of physical activity and increased with physical inactivity regardless of BMI [36]. Both physical inactivity and obesity seem to be strongly and independently associated with diabetes and diabetes-related comorbidities. Physical inactivity is a key contributor to obesity as the excess calories from food and drink are stored as fat. Excess fat is associated with increased insulin resistance. This is especially true when excess fat or excess weight is carried around the abdomen, called central obesity and increases the risk for T2DM.

\section{T2DM and thiamine deficiency}

The hyperglycemia associated with T2DM causes a high cytosolic glucose concentration and accumulation of triosephosphates and leads to intracellular biochemical dysfunction, which can be prevented by disposal of excess triose-phosphates via the reductive pentose-phosphate pathway. The pentose-phosphate pathway is impaired due to thiamine deficiency and correction of thiamine deficiency by thiamine or the thiamine monophosphate prodrug, Benfotiamine, restores disposal of triose-phosphates by the reductive pentose-phosphate pathway in hyperglycemia. The correction simultaneously improves various biochemical dysfunctions including activation of protein kinase $\mathrm{C}$, activation of the hexosamine pathway, increased glycation and oxidative stress, and can halt the development of diabetic nephropathy, neuropathy and retinopathy. The correction of thiamine deficiency by thiamine supplementation also improves $\beta$-cells dysfunction and impaired glucose tolerance and can prevent dyslipidemia and the development of vascular complications in T2DM.

Both thiamine and Benfotiamine can produce reversals of diabetes-induced increased diuresis, hyper-filtration and glycosuria. As documented by various studies, thiamine supplementation may prevent and reverse early-stage diabetic nephropathy [37]. In animal models, thiamine as well as benfotiamine supplementation prevented the development of early-stage nephropathy in diabetic rats and reversed increased urinary albumin excretion in patients with T2DM and microalbuminuria in recent clinical trials [38]. Thus, thiamine and benfotiamine therapy can be a potential novel strategy for the prevention of clinical diabetic nephropathy [39].

\section{Endocrine disruptors and T2DM}

The Progress and the 'Paradox': The prevalence of T2DM, MetS and obesity has dramatically increased during last 3-4 
decades. While lifestyle factors and genetic susceptibility are well-known factors, there is accumulating evidence that the current epidemic of obesity and diabetes is environment-driven and endocrine disrupting chemicals (EDCs) are involved in the pathogenesis. Further, there has been a continuous increase of EDCs and 'obesogens' in the environment and food chains and various obesogens identified so far, appear to have a significant role in the obesity and diabesity pandemic [40]. An ample evidence, both from in vivo and in vitro experimental and epidemiological studies support the hypothesis that exposure to EDCs is related to the current pandemic of T2DM. Thus, while the advances in synthetic chemistry have drastically changed and improved quality of life, the research and studies addressing the role of environmental chemicals in diabetes and obesity suggest that environmental disruption of metabolic processes probably constitutes the 'paradox of progress' [41].

\section{Chronic and acute exposure to EDCs:}

\section{A. Chronic exposure to EDCs}

Both experimental and epidemiological studies underline a role for chronic exposure to low doses of EDCs, which disrupt metabolic processes, skeletal-muscle insulin sensitivity and $\beta$-cell glucose sensing [42]. A number of EDCs have been linked to T2DM including various persistent organic pollutants, arsenic, pesticides and solvents, and other endocrine disrupting chemicals. The sex hormones and other like androgenic chemicals can lead to impaired fasting glucose, glucose intolerance, MetS, T2DM and obesity [43]. Both persistent (PCBs, dioxin, chlorinated pesticides) and nonpersistent (BPA, phthalates) endocrine disruptors are associated with T2DM, impaired fasting glucose, and IR. Endocrine disruptors are also implicated in complications and diseases that relate to T2DM, including atherosclerosis and CVD [44].

Most of EDCs are present in the food chain and accumulate in the fat mass after absorption. The evidence suggests that the relationships between EDCs and dietary metabolic stressors are complex, with an ultimate phenotype that may be exposure-specific [45]. In rodents, bisphenol A influences synthesis and secretion of insulin from $\beta$-cells and disturbs insulin signaling in liver, muscle and adipose tissue through epigenetic changes leading to IR. In humans, epidemiological reports show a statistical link between exposure to pesticides, polychlorinated biphenyl, bisphenol A, phthalates, dioxins or aromatic polycyclic hydrocarbides and heavy metals and T2DM following accidental exposure or lowgrade chronic exposure. There are also various hormonally active chemicals having additive effects and must account for the risk for development of T2DM. The EDCs, as risk factors for T2DM, act at three levels, insulin resistance, obesity, and/or $\beta$-cell function. The duration and intensity of EDCs exposure induces molecular imprinting (epigenetic modifications) on liver, adipose tissue, $\beta$-cells and skeletal muscle. Further, EDCs can lead to ill-effects at low doses on chronic exposure, not predictable by effects at higher doses. Finally, the association between EDCs exposure and diabetes may be due to confounding by fat mass - EDCs are stored in adipose tissue, calorie dense fast foods and beverages from tinned cans containing a large amount of EDCs.

\section{B. Prenatal exposure to EDCs}

The results from animal models have demonstrated that nutritional changes during pregnancy can affect the programming of metabolically active tissues and can induce T2DM in the offspring. The exposure to EDCs in animal models also alters glucose homeostasis in mothers and their offspring when they become adults. The exposure to BPA for a short time during pregnancy may affect the weight and glucose metabolism in offspring later in life, as well as the metabolic state of the mother in the long-term. Similarly, prenatal exposure to tributyltin (TBT) a toxic biocide, in mice promotes adipocyte differentiation that results in increased lipid accumulation and adipose tissue, reduced muscle mass persisting into adulthood and across generations, hyperglycemia with reduced circulating insulin levels accompanied by increased islet apoptosis and reduced cellular proliferation, suggesting a $\beta$-cell defect as a contributing lesion. EDCs can change the expression of genes involved in the control of adipogenesis as well as glucose and lipid metabolism, and the metabolic disorders may not manifest until later in life when the subject is challenged by over-nutrition and lack of exercise. DDT and its metabolites have also been associated with increased risk of higher body weight, insulin resistance; T2D and dyslipidemia in human studies. PBDEs have also been associated with development of fatty liver disease and/or abnormal hepatic lipid metabolism in rodent studies.

\section{Accidental exposures to EDCs}

The data linking EDCs to T2DM come from epidemiological studies following acute and accidental release of EDCs. The study of military personnel exposed to dioxins contained in herbicide orange. The accidental consumption of adulterated rice bran cooking oil in a group of Taiwanese, termed Yu-cheng incident, was related to a higher prevalence of T2DM due to contaminated soil with polychlorinated biphenyl ethers (PCBs), dioxins and furans [46]. Similar observations were related with indirect consumption of persistent organic pollutants (POPs) including organochlorine pollutants, heavy metals and metabolites of the insecticide DDT. The epidemiological studies from non-occupational populations are very suggestive of an independent association between POPs exposure and T2DM.

EDCs, insulin action and glucose disposal: An array of compounds have been documented to impair insulin signal transduction or insulin-stimulated glucose disposal, including tetra-chloro-dibenzo-dioxin (TCDD), tolylfluanid, inorganic and methylated arsenic species, DEHP a phthalate compound, and persistant organic pollutants (POPs). Evidence that EDCs disrupt cellular energy metabolism is supported by data from animal studies. While less specific, a variety of studies have also shown that exposure to various organic and inorganic toxicants promotes global IR or glucose intolerance with altered serum insulin levels. 
While these studies emphasize the diverse array of compounds that can alter insulin action. Atrazine has been shown to promote insulin resistance, an effect exacerbated by a high fat diet [47]. BPA also promotes glucose intolerance and impairs insulin action in a chronic model of exposure coupled with a high fat diet [48]. Interestingly, polychlorinated biphenylsalts, such as, PCB77 and PCB126 are shown to impair glucose tolerance with the induction of systemic insulin resistance when coupled with a low-fat diet [49]. The evidence for the diet-PCB interactions in promoting insulin resistance is complex.

\section{Sex hormones, adiposity and T2DM}

There are important differences in men and women related to incidence as well as the consequences of obesity. The gonadal hormones, androgens and estrogens play a crucial role in shaping such differences. Thus, the two sexes differ in the patterns of fat deposition, fat mobilization and utilization of fat, and the consequences of excess fat stores. Further, there are sexual differences in energy expenditure, or sensory physiology, taste and smell preference, food intake, binge-eating, susceptibility to obesity, responses to leptin-, ghrelin-, or insulin- induced hyperphagia, POMC gene expression in the ARC nucleus, melanocortin system and several other traits. The NPY/AgRP circuit is also sexually dimorphic. Also, NPY immuno reactivity is sexually dimorphic in the ARC, the dorsomedial hypothalamus, and the PVN; NPY-Y1 receptor expression is higher in females compared to males.

The women suffer fewer obesity-related disorders than men do. In fact, they are resistant to free fatty acid-induced insulin release and are therefore less prone to T2DM before menopause but the prevalence of these disorders increases dramatically after menopause [50]. Further, women have an increased responsiveness to leptin and a decreased responsiveness to insulin in comparison to males. These differences are estrogen dependent [51]. The estrogens play a pivotal role by acting directly on the brain or through activation of estrogen receptors (ER) on adipocytes. Further, estrogens protect against increased adiposity/obesity through their effects of suppressing appetite and increasing energy expenditure; estradiol suppresses feeding by enhancing the potency of other anorectic signals (leptin, apolipoprotein, BDNF cholecystokinin) and by decreasing the potency of orexigenic signals such as ghrelin and melanin concentrating hormone [52,53].

\section{Conclusion: Metabolic Response and Adaptive Defence Factors}

For the exogenous insulin therapy to be successful, improving blood nutrient levels is beneficial to vascular health. In case the high dose exogenous insulin therapy is not accompanied with control of blood nutrients, then the IR in the PI3K pathway is not relieved and insulin signaling predominates via the harmful MAPK pathway and increases the risk of vascular events. Thus, going with this hypothesis, the intensive treatment of T2DM with insulin may be harmful in obese patients who are unable to improve lifestyle to achieve negative energy balance. The insulin-responsive tissues, such as the heart, skeletal muscle, adipose tissue, and the liver, are able to protect themselves from nutrient-induced damage by developing IR. Other tissues, such as nerves, eye, kidney and the vasculature, are less protected by IR. Here, the treatment choices would be either to treat the hyperglycemia with higher doses of insulin to override IR but with the risk of increasing insulin-induced metabolic stress in the insulin-responsive tissues [54].

The use of sulfonylureas in such patients may have similar effects. An alternative treatment strategy would be to use nutrient off-loading approaches to lower glucose levels to benefit all tissues, like bariatric surgery, GLP-1 receptor (GLP-1R) agonists, $\alpha$-glucosidase inhibitors, and SGLT2 inhibitors. Metformin is a mild inhibitor of mitochondrial function and activates AMPK in liver and other tissues. The Thiazolidinediones (TZDs) by promoting nutrient partitioning to adipose tissue, cause nutrient off-load effect. Dipeptidyl peptidase-4 (DPP-4) inhibitors do not have nutrient off-loading effects. Thus, the insulin and/or OHA therapy in patients with T2DM must include lifestyle and pharmacological therapies aimed to reverse the excess nutrient imbalance. A regular physical activity helps in controlling body weight, Improves IR and makes muscle cells more sensitive to insulin and to utilize glucose and helps correcting hyperglycemia.The hypotheses, as highlighted by Nolan et al, relating to 'the IR as adaptive defence mechanism' and 'insulin-induced metabolic stress' are attractive concepts and needs to be explored further in-light-of the current research and new studies available.

\section{References}

1. American Diabetes Association (2004) Diagnosis and classification of diabetes mellitus. Diabetes Care 33(1): S62-S69.

2. Galasko G (2015) Looking at diabetes mellitus and its complications: From a different angle.

3. Grundy SM (2004) Definition of metabolic syndrome: report of the national heart, lung and blood Institute/American heart association conference on scientific issues related to definition. Arterioscler Thromb Vasc Biol 24(2): 13-18.

4. Alberti KG (2009) Harmonizing the metabolic syndrome: a joint interim statement of the international diabetes federation task force on epidemiology and prevention; national heart, lung, and blood institute; American heart association; world heart federation; international atherosclerosis society; and international association for the study of obesity. Circulation 120(16): 1640-1645.

5. Aguilar M (2015) Prevalence of the metabolic syndrome in the United States: 2003-2012. JAMA 313(19): 1973-1974.

6. McAllister EJ (2009) Ten putative contributors to the obesity epidemic. Crit Rev Food Sci Nutr 49(10): 868-913.

7. Mahajan R (2017) Insulin resistance: Quest for surrogate markers. Int J App Basic Med Res 7(3): 149.

8. Marroqui L (2014) Nutrient regulation of glucagon secretion: involvement in metabolism and diabetes. Nutr Res Rev 27(1): 48-62.

9. Soeters MR, Soeters P (2012) The evolutionary benefit of insulin resistance. Clin Nutr 31(6): 1002-1007.

10. Poitout V, Robertson RP (2008) Glucolipotoxicity: fuel excess and betacell dysfunction. Endocr Rev 29(3): 351-366. 
11. Nolan CJ, Damm P, Prentki M (2011) Type 2 diabetes across generations: from pathophysiology to prevention and management. Lancet 378(9786): 169-181.

12. Groop PH, Forsblom C, Thomas MC (2005) Mechanisms of disease: pathway-selective insulin resistance and micro vascular complications of diabetes. Nat Clin Pract Endocrinol Metab 1(2): 100-110.

13. Cao W, Ning J, Yang X, Liu Z (2011) Excess exposure to insulin is the primary cause of insulin resistance and its associated atherosclerosis. Curr Mol Pharmacol 4(3): 154-166.

14. Vicent D, Ilany J, Kondo T (2003) The role of endothelial insulin signaling in the regulation of vascular tone and insulin resistance. J Clin Invest 111(9): 1373-1380.

15. Potenza MA, Addabbo F, Montagnani M (2009) Vascular actions of insulin with implications for endothelial dysfunction. Am J Physiol Endocrinol Metab 297(3): E568-577.

16. Hoehn KL, Salmon AB, Hohnen-Behrens C (2009) Insulin resistance is a cellular antioxidant defense mechanism. Proc Natl Acad Sci 106(42): 17787-17792.

17. Saltiel AR, Kahn C (2001) Insulin signalling and the regulation of Insulin signalling and the regulation of glucose and lipid metabolism. Nature 414(6865): 799-810.

18. Gunasekaran U, Gannon M (2011) Type 2 diabetes and the aging pancreatic beta cell. Aging 3(6): 565-575.

19. Barzilai N, Huffman DM, Radhika H (2012) The Critical Role of Metabolic Pathways in Aging. Diabetes 61(6): 1315-1322.

20. Nikhra V (2018) The altered metabolic homeostasis in middle aged and elderly: aging and other determinants of insulin secretion, action and resistance. OAJ Gerontol\& Geriatric Med 3(2): 1-6.

21. Chang AM, Halter JB (2003) Aging and insulin secretion. Am J Physio Endocrinol Metab 284(1): E7-E12.

22. Ramsey KM, Mills KF, Satoh A, Imai SI (2008) Age-associated loss of Sirt1-mediated enhancement of glucose-stimulated insulin secretion in beta cell-specific Sirt1-overexpressing (BESTO) mice. Aging Cell 7(1) 78-88.

23. Cooksey RC, Jouihan HA, Ajioka RS (2004) Oxidative stress, $\beta$-cell apoptosis, and decreased insulin secretory capacity in mouse models of hemochromatosis. Endocrinology 145(11): 5305-5312.

24. Ihm SH, Moon HJ, Kang JG (2007) Effect of aging on insulin secretory function and expression of beta cell function-related genes of islets. Diabetes Research and Clinical Practice 77(3): S150-S154.

25. Eizirik DL, Cardozo AK, Cnop M (2008) The role for endoplasmic reticulum stress in diabetes mellitus. Endocr Rev 29(1): 42-61.

26. Deng Y, Scherer PE (2010) Adipokines as novel biomarkers and regulators of the metabolic syndrome. Ann N Y Acad Sci 1212: E1-E19.

27. Bournat JC, Brown CW (2010) Mitochondrial dysfunction in obesity. Curr Opin Endocrinol Diabetes Obes 17(5): 446-452.

28. Butler AE, Janson J, Bonner-Weir S (2003) $\beta$-Cell deficit and increased $\beta$-cell apoptosis in humans with type 2 diabetes. Diabetes 52(1): 102 110.

29. Kahn SE, Hull RL, Utzschneider KM (2006) Mechanisms linking obesity to insulin resistance and type 2 diabetes. Nature 444(7121): 840-846.

30. Hotamisligil GS, Erbay E (2008) Nutrient sensing and inflammation in metabolic diseases. Nat Rev Immunol 8(12): 923-934.

31. Al Goblan AS, Al-Alfi MA, Khan MZ (2014) Mechanism linking diabetes mellitus and obesity. Diabetes Metab Syndr Obes 7: 587-591.
32. Haffner SM, Stern MP, Hazuda HP (1990) Cardiovascular risk factors in confirmed prediabetic individuals-Does the clock for coronary disease start ticking before the onset of clinical diabetes? JAMA 263(21): 28932898.

33. Ershow AG (2009) Environmental influences on development of type 2 diabetes and obesity: challenges in personalizing prevention and management. J Diabetes Sci Technol 3(4): 727-734.

34. Roden M, Price TB, Perseghin G, Petersen KF, Rothman DL, et al. (1996) Mechanism of free fatty acid-induced insulin resistance in humans. J Clin Invest 97(12): 2859-2865.

35. Eaton SB, Eaton SB (2017) Physical inactivity, obesity and type 2 diabetes: an evolutionary perspective. Res Q Exerc Sport 88(1): 1-8.

36. Sullivan PW, Elaine H, Morrato EH, Wyatt HR, Hill JO (2005) Obesity, inactivity, and the prevalence of diabetes and diabetes-related cardiovascular comorbidities in the US 2000-2002. Diabetes Care 28(7) 1599-1603.

37. Thornalley PJ (2005) The potential role of thiamine (vitamin B1) in diabetic complications. Curr Diabetes Rev 1(3): 287-298.

38. Rabbani N, Thornalley PJ (2011) Emerging role of thiamine therapy for prevention and treatment of early-stage diabetic nephropathy. Diabetes Obes Metab 13(7): 577-583.

39. Babaei JR, Karachalias N, Ahmed N, Battah S, Thornalley PJ (2003) Prevention of incipient diabetic nephropathy by high-dose thiamine and benfotiamine. Diabetes 52(8): 2110-2120.

40. Burgio E, Lopomo A, Migliore L (2015) Obesity and diabetes: from genetics to epigenetics. Mol Biol Rep 42(4): 799-818.

41. Neel BA, Sargis RM (2011) The paradox of progress: environmental disruption of metabolism and the diabetes epidemic. Diabetes 60(7): 1838-1848.

42. Fénichel P, Chevalier N (2017) Environmental endocrine disruptors: New diabetogens? C R Biol 340(9-10): 446-452.

43. Mauvais JF (2018) Gender differences in glucose homeostasis and diabetes. Physiol Behav 187: 20-23.

44. Helsley RN, Zhou C (2017) Epigenenetic impact of endocrine disrupting chemicals on lipid homeostasis and atherosclerosis: a pregnane $\mathrm{X}$ receptor-centric view. Environ Epigenet 3(4)

45. Casals CC, Desvergne B (2011) Endocrine disruptors: from endocrine to metabolic disruption. Annu Rev Physiol 73: 135-162.

46. Wang SL, Tsai PC, Yang CY, Guo YL (2008) Increased risk of diabetes and polychlorinated biphenyls and dioxins: a 24-year follow-up study of the Yucheng cohort. Diabetes Care 31(8): 1574-1579.

47. Lim S, Sun YA, Myung HC, Hak CJ, Kyong SP, et al. (2009) Chronic exposure to the herbicide, atrazine, causes mitochondrial dysfunction and insulin resistance. PLoS One 4(4): e5186.

48. Moon MK, Jeong IK, Jung Oh T, Ahn HY, Kim HH, et al. (2015) Long-term oral exposure to bisphenol A induces glucose intolerance and insulin resistance. J Endocrinol 226(1): 35-42.

49. Baker NA, Karounos M, English V, Fang J, Wei Y, et al. (2013) Coplanar polychlorinated biphenyls impair glucose homeostasis in lean C57BL/6 mice and mitigate beneficial effects of weight loss on glucose homeostasis in obese mice. Environ Health Perspect 121(1): 105-110.

50. Ford ES (2005) Prevalence of the metabolic syndrome defined by the International Diabetes Federation among adults in the U.S. Diabetes Care 28(11): 2745-2749.

51. Clegg DJ (2006) Gonadal hormones determine sensitivity to central leptin and insulin. Diabetes 55(4): 978-987. 
52. Shi H, Seeley RJ, Clegg DJ (2009) Sexual differences in the control of energy homeostasis. Front Neuroendocrinol 30(3): 396-404.

53. Palmer BF, Clegg DJ (2015) The sexual dimorphism of obesity. Mol Cell Endocrinol 402: 113-119.
54. Nolan CJ, Ruderman NB, kahn SE, Pedersen O, Prentki M (2015) Insulin resistance as a physiological defense against metabolic stress: Implications for the management of subsets of Type 2 Diabetes. Diabetes 64(3): 673-686.



Creative Commons Attribution 4.0 International License

For possible submissions Click Here

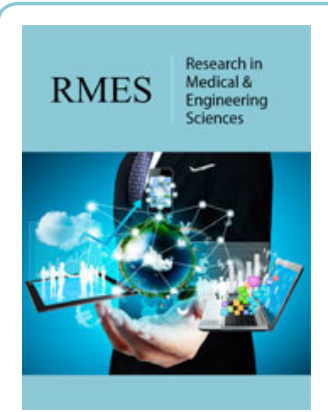

Research in Medical \& Engineering Sciences

\section{Benefits of Publishing with us}

- High-level peer review and editorial services

- Freely accessible online immediately upon publication

- Authors retain the copyright to their work

- Licensing it under a Creative Commons license

- Visibility through different online platforms 